\title{
Value of laparotomy in the diagnosis of obscure gastrointestinal haemorrhage
}

\author{
M P N Lewis, D E Khoo, J Spencer
}

\begin{abstract}
Over a nine year period a total of 137 patients were investigated for obscure gastrointestinal bleeding on one surgical unit. In 20 patients visceral angiography strongly suggested the presence of caecal or right colonic angiodysplasia. These patients were treated by an appropriate colectomy and they are not considered further in this study. Similarly lesions of the small bowel detected by preoperative investigations are not considered here. Fifty five patients were offered diagnostic laparotomy after the failure of other investigations to establish a diagnosis. Two patients refused. A diagnostic laparotomy was performed in the remaining 53. At operation if no visible lesion was seen an on table enteroscopy was performed using a colonoscope passed per oram and, if necessary, per anum. In nine $(17 \%)$ patients no cause for bleeding was found. In $18(34 \%)$ patients there was a small bowel vascular anomaly, in 14 $(26 \%)$ a small bowel tumour, in four (7.5\%) a bleeding Meckel's diverticulum, and in eight $(15 \%)$ other miscellaneous lesions. Laparotomy, with on table enteroscopy where indicated, elucidated the cause of bleeding in 44 patients $(83 \%)$. It was associated, however, with a postoperative death rate of $7 \cdot 5 \%$ (four patients). After seemingly appropriate surgery, rebleeding occurred in 14 patients $(26 \%)$. Of 18 patients with small bowel vascular anomalies seven rebled $(39 \%)$, at an average follow up interval of 32 months.

(Gut 1995; 37: 187-190)
\end{abstract}

Keywords: laparotomy, gastrointestinal haemorrhage.

Gastrointestinal bleeding is a common reason for admission to hospital. In most cases the diagnosis is achieved using upper gastrointestinal endoscopy, colonoscopy, selective mesenteric angiography, scintigraphy, and barium studies. ${ }^{1}$ In a few patients, however, the diagnosis remains concealed despite conventional investigations. These patients have gastrointestinal bleeding of obscure origin. ${ }^{2}$ The decision to proceed to laparotomy after extensive investigation has failed to produce a cause should be based on the knowledge of the diagnostic sensitivity, complication rate, and therapeutic potential of laparotomy with or without on table enteroscopy. This study aims to evaluate the value of laparotomy in obscure gastrointestinal bleeding.

\section{Methods}

During a nine year period between 1982 and 1991,137 patients were referred to one consultant at this institution for the assessment and further treatment of obscure gastrointestinal bleeding. Fifty three patients had a laparotomy to establish a diagnosis after extensive investigations failed to find a cause. Their median age was 62 , with a range of 17 to 84 and comparable sex distribution (male to female ratio 27:26). Most of the patients were extraregional tertiary referrals (42) with a further four from overseas, four from hospitals within our district, and three from our region. All patients had been investigated previously to varying degrees and may therefore be unrepresentative of patients with obscure rectal bleeding in district hospitals. Some of the patients have been reported previously. ${ }^{3-5}$

A full history was taken and a complete physical examination was performed in all patients. Clotting disorders and other blood dyscrasias were excluded by appropriate laboratory tests. Initial investigations included at least one colonoscopy and two or more upper gastrointestinal endoscopies. All patients underwent a selective coeliac, superior and inferior mesenteric angiography. In young patients, a technetium-99m pertechnetate scan was performed to exclude a Meckel's diverticulum. In patients who were not bleeding at the time of angiography in whom no vascular abnormality could be shown, either a ${ }^{99 \mathrm{~m}} \mathrm{Tc}$ labelled red blood cell or ${ }^{99 \mathrm{~m}} \mathrm{Tc}$ labelled colloid scan was performed to aid localisation. Barium contrast studies were always performed in older patients (median age 50). In most this was a small bowel enema, and in patients in whom colonoscopy was incomplete or technically unsuccessful, a barium enema was performed. When a diagnosis could not be reached with these investigations, patients were subjected to laparotomy. When no gross macroscopic abnormality could be found, intraoperative endoscopy was performed using a $160 \mathrm{~cm}$ colonoscope passed per oram and manipulated by the operating surgeon to inspect the upper gastrointestinal tract to the distal ileum.

The resected specimens from these operations were subject to pathological examination and the information gained was used to reach a final diagnosis. When angiodysplasia was suspected the histological specimen was usually injected with intra-arterial barium-gelatin and autoradiography performed for confirmation.

A questionnaire was sent to the patients' referring consultants and general practitioners to determine the longterm outcome of surgery in survivors with respect to rebleeding rates, 
TABLE I Presenting features

\begin{tabular}{lr}
\hline Presenting feature & Number \\
\hline Gastrointestinal bleeding & 32 \\
Melaena & 25 \\
Changed rectal bleeding & 5 \\
Bright rectal bleeding & 1 \\
Haematemesis only & 1 \\
Anaemia (faecal occult blood) & 21 \\
\hline
\end{tabular}

death, and tumour recurrence rates. A response rate of $90 \%$ was obtained. The remainder were followed up for a median duration of six weeks. The average length of follow up in all patients was 31 months (median 24).

\section{Results}

Presenting features (Table I)

Overt gastrointestinal bleeding was the main presenting feature in 35 patients. Eighteen patients presented with melaena only, six with changed rectal bleeding, and four with bright rectal bleeding. Seven had haematemesis in addition to melaena. Three patients presented with shock. Eighteen presented with iron deficiency anaemia only with positive faecal occult blood. The average duration of symptoms was 76 weeks with a range of one week to seven years. The average duration of symptoms varied between final diagnostic groups, being longest paradoxically for small bowel tumours (160 weeks), similarly long for small bowel vascular anomalies (131 weeks), and shortest for Meckel's diverticula (60 weeks). These differences did not reach statistical significance, but nevertheless the duration of symptoms reflects the difficulty in reaching a diagnosis. Sixty per cent of patients presenting with blood in the stool had a small bowel tumour. This was the more probable finding compared with other possible diagnoses $(\mathrm{p}<0.001)$

\section{Investigations}

Selective visceral angiography was carried out in all patients before operation and gastrointestinal contrast studies in $74 \%$. Labelled red cell scans were performed in 14 patients and showed the area of bleeding correctly in six, incorrectly in three. A total of five had a ${ }^{99 \mathrm{~m}} \mathrm{Tc}$ Meckel's scan all of which were negative, including one patient in whom this diagnosis was eventually made.

\section{Operative findings}

At laparotomy the responsible lesion was identified visually in 29 while in a further 15 patients it was identified only after enteroscopy. In nine no lesion was identified despite full operative enteroscopy. Where a lesion was identified resection was performed according to site and suspected pathology. When no lesion could be found a 'best guess' procedure was undertaken bearing angiography and red cell scintigraphy results in mind. In four cases a gastroduodenotomy was also used as bleeding was suspected to originate proximal to the duodenojejunal flexure. These included a partial gastrectomy in one case in whom blood was confirmed to originate from the stomach on laparotomy (histology failed to show any abnormality) and a right hemicolectomy in one in whom there was a high degree of suspicion of angiodysplasia of the caecum. This was not confirmed by histological assessment and there was further occult bleeding in the postoperative period. In one patient the proximal jejunum was found to contain blood in the lumen though no bleeding site could be identified; a resection biopsy specimen of $9 \mathrm{~cm}$ of jejunum failed to show any abnormality. In one patient an old Roux-en-Y anastomosis was excised and refashioned as the suspected bleeding point.

\section{Pathology}

In 44 patients the final diagnosis was confirmed or established by histological examination of resected lesions or by postmortem examination in three of four patients who died (Table II).

\section{Outcome}

There were four deaths in the immediate postoperative period (Table III).

In all these cases laparotomy was undertaken either during or immediately after an acute bleed. In patients two and three the entire bowel was full of blood and no cause of bleeding could be found. The final diagnosis was established at postmortem examination. In case four blood was found in the right hemicolon and a right hemicolectomy was performed, the provisional diagnosis of angiodysplasia being based on the preoperative finding of fresh blood in the proximal colon with a negative selective arteriogram. Histological assessment of the resection specimen did not confirm an angiodysplastic lesion, however, and the patient subsequently bled again. No cause for bleeding was found at postmortem examination. In patients two to four the cause of death resulted from hypovolaemic shock from further uncontrollable blood loss.

There were 14 patients where further gastrointestinal bleeding occurred $(26 \%$, Table

TABLE II Final diagnoses

\begin{tabular}{lc}
\hline Diagnosis & Number \\
\hline Small bowel vascular & 18 \\
Tumour & 14 \\
Leiomyoma & 5 \\
Adenocarcinoma of small bowel & 3 \\
Lymphangioma & 2 \\
Lymphoma of small bowel & 1 \\
Carcinoid & 1 \\
Neurofibroma & 1 \\
Tubular adenoma & 1 \\
Meckel's diverticulum & 4 \\
Miscellaneous & 8 \\
Angiodysplasia caecum & 2 \\
Jejunal diverticulitis & 1 \\
Crohn's disease & $1^{\star}$ \\
Ileal intussusception & 1 \\
Iliacenteric fistula & $1^{\star}$ \\
Gastroduodenal aneurysm & $1^{\star}$ \\
Gastric fundal ulcer & 1 \\
\hline Unknown & 9 \\
\hline
\end{tabular}

^Diagnosis missed on laparotomy. 
TABLE III Postoperative deaths

\begin{tabular}{llll}
\hline & Age & Pathology & Procedure \\
\hline 1 & 66 & Angiodysplasia colon & $\begin{array}{l}\text { Total colectomy+ } \\
\text { ileorectal anastomosis }\end{array}$ \\
2 & 71 & Aneurysm gastroduodenal & None \\
3 & 53 & artery & Iliacenteric fistula \\
4 & 75 & Unknown & $\begin{array}{l}\text { None } \\
\text { Right hemicolectomy }\end{array}$ \\
\hline
\end{tabular}

IV). Seven had a small bowel vascular anomaly (39\% of this group). Three of nine cases in whom no diagnosis had been made experienced further gastrointestinal bleeding $(25 \%)$, which remained undiagnosed though not life threatening. Four of eight miscellaneous cases had further bleeding and only one small bowel tumour (a lymphangioma).

\section{Discussion}

The investigation of gastrointestinal bleeding of longterm duration can present many problems to the surgeon. Not least is the course of action after failure of localisation procedures to elucidate a cause. This has been previously reported in $16 \%$ of patients at this institution. ${ }^{5}$ In this series, the personal experience of one surgeon (JS), showed 53 of 137 $(41 \%)$ of all patients investigated for obscure intestinal bleeding required a laparotomy for the final diagnosis.

Before the advent of angiography and the routine use of endoscopy the sensitivity of laparotomy for gastrointestinal bleeding was low. A study by Retzlaff in 1961 of 100 cases found that in 53 no diagnosis was obtained. ${ }^{6}$ More recently with the introduction of more sensitive investigations the preoperative diagnostic yield has been higher. ${ }^{1578}$ Fibreoptic jejunoscopy is gradually finding a use in obscure gastrointestinal bleeding with encouraging initial studies. ${ }^{9-13}$ It appears to have a sensitivity of between 33 to $70 \%$. This investigation has recently been introduced to this hospital, though its fairly esoteric benefit and high cost probably preclude its use in most units.

A diagnostic laparotomy will still be required for the small number of obscure bleeders of longterm duration. There is a definite need for on table enteroscopy in these cases by whichever method. ${ }^{1415} \mathrm{We}$ have used a colonoscope with a working length of $160 \mathrm{~cm}$, which is passed per oram through the pylorus when it is then negotiated past the duodenojejunal flexure. The small bowel is then examined segmentally with an occlusive clamp applied lightly at appropriate distances to prevent distension of the distal bowel. In this series, $34 \%$ of those in whom a diagnosis was established at laparotomy, the lesion was found only with enteroscopy. Its value has been confirmed previously. ${ }^{314}$ Certainly in this series only $55 \%$ of cases had diagnosis found by visual inspection or manual palpation of the bowel. Despite a diagnostic yield of $83 \%$, however, $26 \%$ experienced further gastrointestinal bleeding over an average period of 31 months. This was especially seen in cases of small bowel arteriovenous malformations where $39 \%$ rebled, an experience borne out by others. ${ }^{11} 1516$ It might be asked whether small bowel vascular anomalies reflect a disease of the entire small bowel rather than discrete lesions. In most cases there is more than one lesion identified at enteroscopy. At present it is our policy to resect all enteroscopically visible lesions. When blood is found predominantly in the colon on table colonic lavage and intraoperative colonoscopy has been advocated ${ }^{17}$ though in our series there was only one patient with active colonic bleeding at time of operation. We were satisfied that preoperative colonoscopy and angiography excluded colonic bleeding in all others.

There is a need to diagnose small bowel tumours early and surgical resection carries a good outcome for benign tumours. ${ }^{18}$ Adenocarcinoma of the small bowel is usually found at a clinically advanced stage because of the difficulty of diagnosis. Resection is more effective than chemotherapy though the prognosis is poor. ${ }^{19}$

Laparoscopy has been advocated as a tool for the investigation of gastrointestinal haemorrhage. ${ }^{20}$ However, its sensitivity for small vascular lesions is probably low. Additionally some small bowel tumours can only be located by careful palpation of the bowel. We have recently found it useful for the location and resection of more obvious tumours and Meckel's diverticula. In our series, and in others, small bowel tumours have been present for a long time before resection. Early laparoscopy would exclude the presence of most of these tumours, and permit more leisurely investigation of the chronic bleeding patient at less risk. Alternatively, if appropriate, the surgeon may proceed at once to laparotomy and on table enteroscopy if laparoscopy failed to identify the cause. The negative laparotomy rate in our series was $17 \%$. Of these $33 \%$ experienced further bleeding despite our policy of 'best guess' resection. There may be a case for split ileostomy in a proportion of these difficult cases.

The postoperative death rate of $8 \%$ is high compared with other death rates for investigative laparotomy and can be explained by the three patients in whom postoperative bleeding was massive and uncontrolled. If possible laparotomy is best performed when the patient is stable. These deaths reflect the worse outlook in the more acute and faster bleeding patient.

Laparotomy should be considered in cases of obscure gastrointestinal bleeding of longterm duration, and as small bowel tumours form a high proportion this should be done sooner rather than later. When flexible jejunoscopy is available, however, it should be

TABLE IV Rebleeding rate by diagnostic group

\begin{tabular}{lcl}
\hline Diagnostic group & Number & $\begin{array}{l}\text { Further bleeding } \\
\text { (number and rate) }\end{array}$ \\
\hline Meckel's diverticulum & 4 & 0 \\
Small bowel vascular & 18 & $7(39 \%)$ \\
Small bowel tumour & 14 & $1(7 \%)$ \\
Undiagnosed & 9 & $3(33 \%)$ \\
\hline
\end{tabular}


considered first. A laparoscopy may precede laparotomy to exclude obvious lesions such as a small bowel tumour or Meckel's diverticulum, which may be removed through a minimal incision. If, at laparotomy, palpation and visual inspection of the small bowel fails to show a bleeding lesion, then on table enteroscopy should be performed.

1 Lau WY, Fan ST, Wong SH, Wong KP, Poon GP, Chu $\mathrm{KW}$, et al. Preoperative and intraoperative localisation of 869-77.

2 Birke G, Engstedt L. Melaena and haematemesis - followup investigation with special reference to bleeding of unknown origin. Gastroenterologia 1956; 85: 97-115.

3 Desa LA, Ohri SK, Hutton KA, Lee H, Spencer J. Role of intraoperative enteroscopy in obscure gastrointestinal bleeding of small bowel origin. Br F Surg 1991; 78: 192-5.

4 Thompson J, Hemingway A, McPherson G, Rees H, Allison $D$, Spencer J. Obscure gastrointestinal haemorrhage of small bowel origin. BMF 1984; 288: 1663-5.

5 Thompson $N$, Salem RR Hemingway AP, Rees HC, Hodgson $\mathrm{HJF}$, Wood CB, et al Specialist investigation of Hodgson HJ, Wood CB, et al. Specialist investigation of

obscure gastrointestinal bleeding. Gut 1987, 28: 47-51. 6 Retzlaff J, Hagedorn A, Bartholomew L. Abdominal exploration for gastrointestinal

7 Moncure A, Tompkins R, Athanasoulis C, Welch C. Occult gastrointestinal bleeding: newer techniques of diagnosi and therapy. Adv Surg 1989; 22: 141-78.

8 Spiller RC, Parkins RA. Recurrent gastrointestinal bleeding of obscure origin: report of 17 cases and a guide to logical management. Br f Surg 1983; 70: 489-93.

9 Tada M, Kawai K. Small bowel endoscopy. Scand $\mathcal{f}$ Gastroenterol 1984; 19: 39-52.

10 Lewis B, Waye J. Chronic gastrointestinal bleeding of obscure origin: role of small bowel enteroscopy. Gastroenterology 1988; 94: 1117-20.

11 Szold A, Katz LB, Lewis BS. Surgical approach to occult gastrointestinal bleeding. Am $\mathcal{F}$ Surg 1992; 163: 90-2.

12 Morris A, Wasson L, MacKenzie J. Small bowel enteroscopy in undiagnosed gastrointestinal blood loss. Gut 1992; 33: 887-9.

13 Van GA, el Nawar A, Adler M, Cremer M. Enteroscopy: methods and results. Acta Gastroenterol Belg 1992; 55: 169-75.

14 Flickinger E, Stanforth A, Sinar D, MacDonald K, Lannin $\mathrm{D}$, Gibson J. Intraoperative video panendoscopy for diagnosing sites of chronic intestinal bleeding. Am 7 Surg nosing sites of chro

15 Rees AM, Benacci JC, Sarr MG. Efficacy of intraoperative enteroscopy in diagnosis and prevention of recurrent, occult gastrointestinal bleeding. Am f Surg 1992; 163 94-8.

16 Lewis B, Kornbluth A, Waye J. Small bowel tumours: yield of enteroscopy. Gut 1991; 32: 763-5.

17 Scott HJ, Lane IF, Glynn MJ, Thoedorou NA, LloydDavies E, Reynolds KW, et al. Colonic haemorrhage: technique for rapid intra-operative bowel preparation and colonoscopy. Br ₹ Surg 1986; 73: 390-1.

18 Desa L, Bridger J, Grace P, Krausz T, Spencer J. Primary jejunoileal tumours: a review of 45 cases. World 7 Surg 1991; 15: 81-7.

19 Lioe T, Biggart J. Primary adenocarcinoma of the jejolunum and ileum: clinicopathological review of 25 cases. $\mathcal{F}$ Clin Pathol 1990; 43: 533-6.

20 Sackier J. Laparoscopy in the emergency setting. World $\mathcal{f}$ Surg 1992; 16: 1083-8. 